\title{
Chapter 10. Diagnostic procedure for CKD
}

(C) Japanese Society of Nephrology 2009

- Diagnosis and staging of CKD is made based on its definition.

- After diagnosis of CKD stage, primary disease and background factors are sought.

- In order to search for primary disease and background factors, physical examination and medical interview are useful and essential.

\section{Treatment plans for each stage of CKD (Table 10-1)}

A high-risk group for CKD

- In cases with normal kidney function (GFR $\geq 90 \mathrm{~mL} /$ $\min / 1.73 \mathrm{~m}^{2}$ ) and a risk factor for CKD (Table 10-2), regular urinalysis follow-up (preferably urinary albumin to creatinine ratio in a diabetic) is recommended.

\section{CKD stages 1 and 2}

- These stages represent a condition where kidney function is normal (GFR $\geq 90 \mathrm{~mL} / \mathrm{min} / 1.73 \mathrm{~m}^{2}$ ) or mildly low $\left(60 \leq \mathrm{GFR}<90 \mathrm{~mL} / \mathrm{min} / 1073 \mathrm{~m}^{2}\right)$ and kidney damage ( $\approx$ proteinuria) exists.

- There are two categories of CKD. One includes kidney disease in a narrow sense, which is indicated for renal biopsy such as kidney diseases caused by glomerulonephritis, interstitial nephritis, vasculitis, collagen disease, etc. The other includes other CKD associated primarily with lifestyle-related disease or aging.

- In a case of possible glomerulonephritis or kidney disorder related to collagen disease, an individual is referred without delay to nephrologists for establishing a diagnosis and initiating treatment of the primary disease. A patient with nephrotic syndrome or massive proteinuria needs prompt treatment by nephrologists.

- Lifestyle-related disease such as hypertension or diabetes is not always indicated for renal biopsy in most cases even though abnormal urinalysis persists. Primary care physicians play an important role for these diseases. They are required to treat the disease with a goal of preserving kidney function and reducing risk for CVD while fully intervening in lifestyle-related disease. They collaborate with nephrologists as needed.

CKD stages 3 and 4

- Stage 3 represents mildly reduced kidney function $\left(30 \leq \mathrm{GFR}<60 \mathrm{~mL} / \mathrm{min} / 1.73 \mathrm{~m}^{2}\right)$, while stage 4 represents severely reduced kidney function $(15 \leq$ GFR $<30 \mathrm{~mL} / \mathrm{min} / 1.73 \mathrm{~m}^{2}$ ).

- A stage 3 patient is treated in cooperation with nephrologists after consultation, while stage 4 is treated by nephrologists. At stage 3, progression to ESKD is accelerated and the risk for CVD development is significantly increased. An attending physician is careful about acute decline in kidney function caused by nephrotoxic agents such as NSAIDs and certain antibiotics or by dehydration.

The point of medical interview and physical examination for consultation on CKD

History taking on a CKD patient (see the checklist)

- Past history: it is important to take a history of potential primary disease of CKD. If a patient does not 
Table 10-1 CKD staging and treatment plan

\begin{tabular}{|c|c|c|c|}
\hline CKD stage & Severity & $\mathrm{eGFR}\left(\mathrm{mL} / \mathrm{min} / 1.73 \mathrm{~m}^{2}\right)$ & Plan \\
\hline- & High risk & $\geq 90$ (risk factors of CKD) & $\begin{array}{l}-\mathrm{CKD} \text { screening } \\
-\mathrm{CKD} \text { risk reduction }\end{array}$ \\
\hline 1 & $\begin{array}{l}\text { Kidney damage }+ \\
\text { Normal or increased GFR }\end{array}$ & $\geq 90$ & $\begin{array}{l}\text { Add on the above } \\
\text {-Diagnosis and treatment of CKD } \\
\text { - Treat comorbid conditions } \\
\text {-Retard the progression of CKD } \\
\text {-CVD risk reduction }\end{array}$ \\
\hline 2 & $\begin{array}{l}\text { Kidney damage }+ \\
\text { Decreased GFR, mild }\end{array}$ & $60-89$ & $\begin{array}{l}\text { Add on the above } \\
\text {-Evaluate the progression rate }\end{array}$ \\
\hline 3 & Decreased GFR, moderate & $30-59$ & $\begin{array}{l}\text { Add on the above } \\
\text {-Evaluate and treat CKD-related complication } \\
\text { (anemia, hypertension, secondary hyperparathyroidism, etc.) }\end{array}$ \\
\hline 4 & Decreased GFR, severe & $15-29$ & $\begin{array}{l}\text { Add on the above } \\
\text {-Prepare for dialysis/transplantation }\end{array}$ \\
\hline 5 & Kidney failure & $<15$ & -Start dialysis or transplant (for uremic symptoms) \\
\hline
\end{tabular}

Table 10-2 Risk factors of development and progression of CKD

- Hypertension

- Impaired glucose tolerance, diabetes mellitus

- Obesity, dyslipidemia, metabolic syndrome (lifestyle-related disease)

- Collagen disease, systemic infection

- Urinary tract stone, urinary tract infection, prostate hypertrophy

- Family history of CKD, low birth weight

- Past history of screening (urine tests, kidney function, kidney size and shape)

- Habitual drugs (NSAIDs), supplements

- Past history of acute kidney failure

- Smoking

- Elderly

- Single kidney, kidney atrophy (small kidney)

mention this voluntarily, a physician asks by naming specific diseases including kidney disease, diabetes, hypertension, urinary tract infection, especially reflux nephropathy due to vesicoureteral reflux (VUR), and atherosclerotic disease such as cerebrovascular disease, coronary artery disease, and peripheral artery disease. If there is a history of these diseases, disease duration has to be confirmed. It is necessary to confirm if a patient has a history of chronic painful disease such as chronic headache, rheumatoid arthritis, and dysmenorrhea because these diseases have a connection with excessive analgesic use which may injure the kidney.

- History of health examination (history of urinary examination): a diagnosis of primary disease can be narrowed down from a history of abnormal urinary finding, time of development (age), and so on. School-based or workplace-based urinary examination might have been done depending on a patient's position in society.

- Gross hematuria, urine volume, urinary features: patients may have previously noticed gross hematuria despite mild hematuria or proteinuria in the current urinalysis. In such cases, it should be confirmed with patients whether they have a history of upper respiratory tract infection or intestinal tract infection prior to gross hematuria. IgA nephropathy is known to be associated with gross hematuria following the above infections. Acute nephritic syndrome is also suspected when urinary abnormalities including hematuria, edema, and hypertension emerge at 2-3 weeks after upper respiratory tract infection. A change of urine volume needs to be asked. In some cases of advanced proteinuria, urine appears foamy, which is helpful for estimating the time of its development.

- History of pregnancy: a female patient has to be asked if she has a history of pregnancy-induced hypertension. Specific questions are asked such as urinary abnormalities during pregnancy and after delivery, hypertension, and edema.

- Family history: primary disease may be guessed from family history of kidney failure, kidney disease or genetic disease such as Alport syndrome, polycystic kidney disease, familial nephritis, and Fabry disease. Family history of hypertension, diabetes, hyperuricemia, and metabolic syndrome that can be a background factor of CKD is helpful for evaluation of risks.

- Past laboratory data: as much information as available of changes in kidney functions in the past is useful for predicting future progression of CKD. 
- Lifestyle: smoking is a risk factor for progression of CKD, so its history should always be taken. Alcohol intake easily causes dehydration if habitual and can be a background factor for hyperuricemia also, so it needs to be confirmed. It is important to know situations with regard to physical exercise when a urine specimen is collected because hard exercise may cause abnormal results of urinalysis. It is important to take history of health food or supplement intake or folk remedies such as herbal medicines.

- History of drugs, history of exposure to substance toxic to the kidney: it is important to take a history of intake of the following agents at the first examination: over-thecounter drugs, especially antipyretic-analgesics, active vitamin $\mathrm{D}$, calcium-containing agents, antihypertensive agents, especially ACE inhibitors and ARBs that may cause kidney injury or reduced kidney function.

\section{The point of physical examination in CKD management}

- Vital signs: body weight, blood pressure, body build (obesity-related nephropathy), urinary output, and level of consciousness.

- Head, neck, and eyes: anemia, jaundice, blepharal edema, ocular fundus (diabetic retinopathy, hypertensive retinopathy, arteriosclerosis, and cholesterol embolus), corneal/lens opacity (Fabry disease), conical cornea (Alport syndrome). Ears: hearing loss (Alport syndrome, adverse effects of aminoglycoside antibiotics). Oral cavity: macroglossia (amyloidosis), tonsillar hypertrophy, fur (IgA nephropathy, streptococcal infection), cervical vein dilatation, collapse (assessment of body fluid), bruit over the neck (atherosclerosis).

- Chest: signs of heart failure (heart murmurs, pulmonary edema, pleural fluid), pulmonary alveolar hemorrhage, epicarditis (SLE, uremia).

- Abdomen: bruit (renal artery stenosis), palpable kidney (polycystic kidney), tap pain over the kidney (acute pyelonephritis, renal infarction), abdominal pain (Henoch-Schönlein purpura, cholesterol embolus).

- Prostate gland: hypertrophy (urinary obstruction, postrenal acute renal failure).

- Extremities: edema (body fluid retention), arthralgia or joint deformity (gout, rheumatoid arthritis, collagen disease, Henoch-Schönlein purpura), blue toe (cholesterol embolus), pains (Fabry disease).

- Skin: poor turgor (dehydration), purpura (HenochSchönlein purpura), livedo reticularis (reticular rash: cholesterol embolus, vasculitis), angiokeratoma/acroparesthesia/anhidrosis (Fabry disease). 